\title{
Embryo biopsy and development: the known and the unknown
}

\author{
Federica Zacchini ${ }^{1}$, Roberta Arena ${ }^{1}$, Adam Abramik ${ }^{1}$ and Grazyna E Ptak ${ }^{1,2,3}$ \\ ${ }^{1}$ Institute of Genetics and Animal Breeding, Polish Academy of Sciences, Jastrzebiec, Poland, ${ }^{2}$ Faculty of Veterinary \\ Medicine, University of Teramo, Teramo, Italy and ${ }^{3}$ Malopolska Center of Biotechnology, Jagiellonian University, \\ Kraków, Poland
}

Correspondence should be addressed to F Zacchini; Email: f.zacchini@ighz.pl

\begin{abstract}
Preimplantation genetic diagnosis (PGD) has been introduced in clinical practice as a tool for selecting 'healthy' embryos before their transfer in utero. PGD protocols include biopsy of cleaving embryos (blastomere biopsy (BB)) or blastocysts (trophectoderm biopsy (TB)), followed by genetic analysis to select 'healthy' embryos for transfer in utero. Currently, TB is replacing the use of BB in the clinical practice. However, based on the European Society of Human Reproduction and Embryology Preimplantation Genetic Diagnosis Consortium reports, BB has been used in $>87 \%$ of PGD cycles for more than 10 years. An exhaustive evaluation of embryo biopsy (both BB and TB) risks and safety is still missing. The few epidemiological studies available are quite controversial and/or are limited to normalcy at birth or early childhood. On the other hand, studies on animals have shown that BB can be a risk factor for impaired development, during both pre- and postnatal life, while little is known on TB. Thus, there is an urgent need of focused researches on $\mathrm{BB}$, as it has contributed to give birth to children for more than $\mathbf{1 0}$ years, and on TB, as its application is significantly growing in clinical practice. In this context, the aim of this review is to provide a complete overview of the current knowledge on the short-, medium- and long-term effects of embryo biopsy in the mouse model.

Reproduction (2017) 154 R143-R148
\end{abstract}

\section{General overview}

Since the 90s, PGD has been offered to couples undergoing assisted reproduction. In PGD, embryos produced by in vitro fertilization or intracytoplasmatic sperm injection are screened for genetic defects, allowing only 'healthy' embryos to be selected for transfer in utero. One component of PGD protocol is embryo biopsy to obtain one or few cells to be genetically screened. Embryo biopsy can be performed on cleaving embryos (blastomere biopsy (BB), consisting in the removal of one or two blastomere from 8-cell embryos) or on blastocyst stage embryos (trophectoderm biopsy - TB, consisting of the removal of few cells from trophectoderm). Based on the ESHRE Preimplantation Genetic Diagnosis Consortium reports (Harper et al. 2012, Montou et al. 2014, De Rycke et al. 2015), BB has been used in the majority of PGD cycles (BB $>87 \%$ vs $\mathrm{TB}<1 \%$ ) for over 10 years. However, in the last years, the use of TB is replacing that of BB in the clinical practice.

Since genetic testing of a single cell obtained by early embryo biopsy was shown to be possible (Monk et al. 1987), much attention has been devoted to optimizing the technique to improve embryo survival. To this aim, Takeuchi et al. (1992) compared three different protocols - enucleation, aspiration and extrusion - for the removal of one blastomere from a developing embryo, showing that the aspiration method was the less harmful protocol for $\mathrm{BB}$ when the procedure was performed on both 4- and 8-cell stage embryos. Pierce and coworkers reported that 8 -cell stage embryos were less sensitive to the procedure than 4-cell ones (Pierce et al. 1997). Concerning TB, Carson and coworkers showed that the excision of herniating trophectoderm after hatching was the safest way to perform blastocyst biopsy compared to the aspiration method (Carson et al. 1993).

First reports in 90s, concerning development to blastocyst stage and the rate of cell division after BB (Wilton \& Trounson 1989, Takeuchi et al. 1992, Pierce et al. 1997) as well as embryo survival following TB (Carson etal. 1993), were quite reassuring. More recently, time-lapse analysis revealed impaired developmental dynamic in both human and mouse BB-embryos, in terms of timing of blastulation, expansion/contraction movements and hatching (Duncan et al. 2009, Terada et al. 2009, Ugajin et al. 2010, Bar-el et al. 2016, Cimadomo et al. 2016, Yao et al. 2016). No information on developmental dynamic following TB has been published so far.

Molecular analyses on mouse embryos developed after BB, but not TB, have been carried out by few researchers (Wu et al. 2014, Zacchini et al. unpublished). These studies showed deregulated expression of genes involved in DNA methylation, chromatin remodeling, 
cell cycle and differentiation, oxidative stress, metabolism (Wu et al. 2014) and of three imprinted genes UBE3A, SNRPN and PEG1 (S Sampino, F Zacchini, GE Ptak, unpublished observation) in $4.5 \mathrm{dpc}$ blastocysts. Epigenetic analysis of $7.5 \mathrm{dpc}$ embryos, following BB, revealed a reduced global methylation profile vs that of control embryos. In particular, 20 differentially methylated loci were identified in the BB embryo group, 11 of which involved in important developmental events (i.e. cell proliferation and differentiation) or the onset of developmentally related diseases (i.e. cardiovascular disease and infertility) (Wu et al. 2014).

Regarding the effects of BB and TB on the offspring's further development, epidemiological studies are quite controversial and/or are limited to normalcy at birth or early childhood (Banerjee et al. 2008, Middelburg et al. 2011, Desmyttere et al. 2012, Thomaidis et al. 2012, Winter et al. 2015, Cimadomo et al. 2016, Sunkara et al. 2016). Though the majority of studies did not show an increased incidence of pregnancy complications following embryo biopsy, some reported that BB is associated with reduced implantation rates, increased risks of low birth weight or preterm birth (Banerjee et al. 2008, Thomaidis et al. 2012, Cimadomo et al. 2016) and, in early childhood, with impaired cognitive and/or motor development (Banerjee et al. 2008, Middelburg et al. 2011, Thomaidis et al. 2012, Cimadomo et al. 2016), while no indication on TB pregnancy/babies are available. Research on mouse model has shown that BB is not only associated with increased pregnancy loss and reduced/delayed fetal development, but also with impaired placental functions and epigenetic defects in both fetuses and placentae (Sugawara et al. 2012, Yang et al. 2012, Sato et al. 2014, Wu et al. 2014, Yao et al. 2016). Regarding TB, research on mouse model is very limited so far. Gentry and Critser (1995) reported neither pregnancy complication nor reduced birthweight after TB. More recently, Taskin and coworkers described that, even if blastocyst viability was not affected, TB was associated with reduced implantation rate and development to term (Taskin et al. 2016). Additional studies are necessary to confirm whether (or not) TB may affect post implantation development.

Concerning the postnatal development of mice obtained following $\mathrm{BB}$ and $\mathrm{TB}$, the first reports were again quite reassuring, as they did not show any adverse effects of both procedures on the offspring at birth (i.e. congenital defects, developmental delays or reduced body weight) or general health in adulthood (i.e. body weight, blood parameters or gross morphology of major internal organs) (BB: Cui et al. 1993, 1994; TB: Gentry \& Critser 1995). However, in the last decade, different research groups have described increased body weight/size and impaired behavior in BB mice and have suggested reduced fertility in adult females (Yu et al. 2009, Zeng et al. 2013, Zhao et al. 2013, Sampino et al. 2014, Wu et al. 2014).
Studies to assess epigenetic, behavioral, reproductive and/or metabolic health in the pre- or postnatal life following TB have not been published so far, while few research groups have performed studies to assess BB risk(s) and safety (Yu et al. 2009, 2013, Sugawara et al. 2012, Yang et al. 2012, Zeng et al. 2013, Zhao et al. 2013, Sampino et al. 2014, Sato et al. 2014, Wu et al. 2014). Thus, in the following sections, we will focus our attention on BB, rather than TB.

\section{Effects of blastomere biopsy on feto-placental development}

A gross morphological evaluation of developing mouse fetuses obtained following BB did not show delayed development in early pregnancy $(7.5 \mathrm{dpc}$, Yang et al. 2012), while reduced fetal, but not placental, weight was observed starting from $12.5 \mathrm{dpc}$ (Sato et al. 2014, Yao et al. 2016). Of relevance, a low fetal/placental weight ratio was observed in BB conceptuses, suggesting increased risk of cardiovascular diseases later in life (Thornburg et al. 2010). Although placental gross morphology and weight seem not to be affected by BB, impaired placental functions have recently been described (Sugawara et al. 2012, Sato et al. 2014, Yao et al. 2016). Sugawara and coworkers showed alterations in steroid metabolism, such as increased progesterone levels and impaired steroid clearance/elimination, in placentae and fetal livers (Sugawara et al. 2012). Compromised steroid metabolism has been associated with (i) defective placental vasculogenesis, which may affect nutrient supply to the fetus; (ii) defective signaling for the induction of labor and (iii) delayed fetal development, resulting in low birth weight and indicative of a higher predisposition to cardiovascular disease later in life. Other studies have reported deregulation of STAT, SOCS and MMP signaling pathways, alteration of oxidative stress, inflammation and impaired expression of imprinted genes (Igf2pO, H19 and lgf2r) in full-term mouse placentae (Sato et al. 2014, Yao et al. 2016). Altogether, these data suggest that BB may be a risk factor for defective placental vascular development and function, as well as intrauterine growth restriction and obstetric complications (i.e. premature membrane rupture and dysfunctional labor).

\section{Reproductive fitness in mice conceived following blastomere biopsy}

No effect of BB was described with regard to pregnancy rate and litter size when comparing BB mice vs control(s) at reproductive age (Sampino et al. 2014, Yu et al. 2013). However, Yu and coworkers showed that aged (i.e. 40-week-old) females, born following $\mathrm{BB}$, had reduced pregnancy rates, numbers of developing follicles and ovarian weights, in addition to altered steroid levels (Yu et al. 2013). To investigate 
the underlying mechanism(s) responsible for reduced ovarian function, proteomic analyses of ovarian tissues from pubertal (10-week-old) and aged (40-week-old) females were performed. These analyses showed that ovarian tissues from both BB-pubertal and BB-aged females were characterized by impaired expression of three proteins (HSPA4, PSMB8 and ALDH1A1) associated with ovarian development, follicle formation and could indicate a higher risk of ovarian cancer. Further analyses are necessary to confirm this study and to evaluate whether reproductive function could also be impaired in male BB subjects.

\section{Neurodevelopmental disorders induced by blastomere biopsy}

In the last decade, a few research groups have investigated whether BB could be associated with an increased risk of neurodevelopmental disorders (Yu et al. 2009, Zeng et al. 2013, Zhao et al. 2013, Sampino et al. 2014, Wu et al. 2014). Behavioral tests showed that mice born following BB had impaired memory and motor coordination, increased anxiety and altered responses to threatening or stress stimuli. Of relevance, these studies showed an increased predisposition to behavioral anomalies in male subjects, but not in females. To clarify the mechanism behind the impaired behavior and stress response, the brain and adrenal glands (peripheral organs) were selected as target organs. Histological and ultrastructural examination of the brain revealed structural abnormalities in the corpora striatum and hippocampus, such as hypomyelination, axonal swelling, neuronal regression and mitochondrial degeneration (Yu et al. 2009, Wu et al. 2014). Proteomic analysis of brain tissues from BB mice identified 36 differentially expressed proteins, 17 of which are associated with neurodegenerative diseases, including Alzheimer's and Parkinson's disease (Yu et al. 2009). Epigenetic analysis of brain tissues showed reduced methylation and increased hydroxymethylation. In particular, methylated DNA immunoprecipitation (MeDIP) analysis identified 11 differentially methylated loci, six of which are associated with neural disorders (Wu et al. 2014). Furthermore, Zhao and coworkers showed that impaired behavior in BB mice was associated with epigenetic defects in brain tissues, while the same association was not observed in control mice (Zhao et al. 2013). Regarding the adrenal glands, Zeng and coworkers described an altered response to cold stress in BB mice, which was associated with impaired structure and function of this periferical organ, and not with anomalies in the hypothalamus (Zeng et al. 2013). Altogether, these studies suggest that BB may be responsible for a higher incidence of altered behavior in offspring and could predispose them to neurodevelopmental and psychiatric diseases.

\section{How embryo biopsy may affect embryo development}

The preimplantation period is a short window of mammalian development characterized by a welldefined sequence of programming events that will decide the future of the developing embryo. In this period, epigenetic and/or metabolic changes (i.e. adaptations), occurring to promote survival in an inadequate environment (e.g. a suboptimal culture system, maternal undernutrition), may cause permanent changes to developmental programming, thus leading to increased risk of developmental defects and/or metabolic disorders (Barker 2007).

With regard to blastomere biopsy, no research has been carried out to investigate whether or how the removal of one cell from a developing embryo could trigger adaptations that persist further in development (both pre- and post-natally). However, based on the current knowledge in the field of developmental and reproductive biology, we can speculate that:

- The removal of one blastomere deprives the embryo of important developmental determinants necessary to produce a healthy individual. This speculation is based on different studies showing that embryos at early stages of development (including at the 4-cell stage) are characterized by individual blastomeres that differ in their developmental fate and potency (PiotrowskaNitsche et al. 2005, Tarkowski et al. 2010), as well as in their transcriptomic profile and epigenetic features (Torres-Padilla et al. 2007, Tang et al. 2011). In this context, BB may be responsible for the embryo's adaptations that occurred as a consequence of blastomere/determinant removal.

- BB acts as a 'stress stimulus' that interferes with the proper sequence of events necessary for successful development. As widely described in human and animal models, a suboptimal preimplantation environment (such as in vitro culture) can perturb proper epigenetic remodeling or critical transcriptional events during early development (Thompson et al. 2006, Lucas 2013), resulting in impaired fetoplacental and postnatal development. With respect to $\mathrm{BB}$, there is limited knowledge of epigenetic or transcriptional perturbation in early embryos (Wu et al. 2014); however, due to defects observed in BB conceptuses and offspring (Sugawara et al. 2012, Zhao et al. 2013, Wu et al. 2014), it is plausible that the removal of one blastomere from the developing embryo perturbs the proper sequence of developmental programming events.

With regard to trophectoderm biopsy, there is no extensive knowledge concerning detrimental effects (if any) on both pre- and postnatal life. However, as the procedure is carried out at the end of preimplantation 
window, it could be speculated that the removal of few cells for trophectoderm could affect proper placental development and function. If so, impaired placentation could also perturb both fetal and offspring development. Much effort must be done to assess potential risk (if any) of TB.

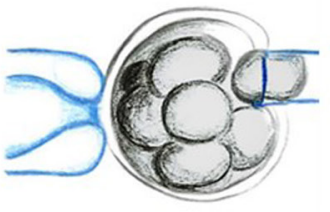

Blastomere Biopsy
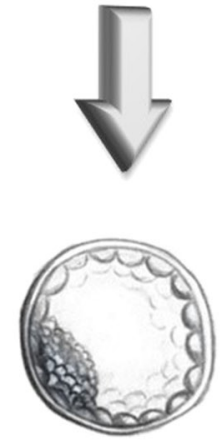

Blastocyst stage

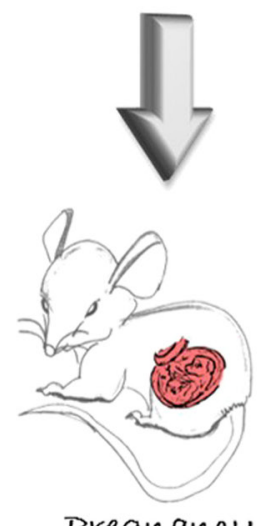

Pregnancy

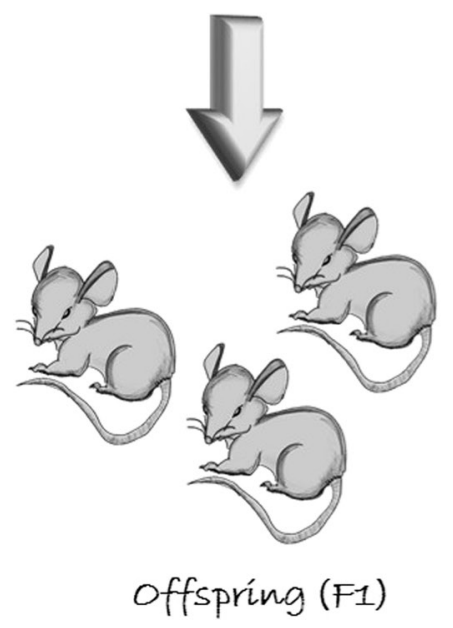

Developmental Defects Observed after Blastomere Biopsy

\section{Preimplantation period}

- Delayed blastulation

- Defective hatching

- Abnormal contraction/expansion

- Reduced cell number (mainly in $I C M$ )

- Deregulated expression of genes involved in a variety of biological processes (i.e. DNA methylation, metabolism, embryo development)

\section{Post-implantation period}

- Reduced survival rate

- Low fetal weight

- Low fetal/placental ratio

- Epigenetic defects in fetuses and placentae

- Impaired placental function

\section{Postnatal period}

- Increased body weight (mainly in males)

- Impaired memory

- Impaired locomotor coordination

- Increased anxiety

- $\quad$ Altered response to stress

- Structural abnormalities in the corpora striatum and hippocampus (i.e. hypomethylation, axonal swelling, neuronal regression, mitochondrial degeneration)

Epigenetic and proteomic defects in brain

- Impaired function of adrenal glands

- Reduced fertility in 40-week-old females

\section{References}

Duncan et al., 2009; Terada et al., 2009, Ugajin et al., 2010; Sugawara et al., 2013; Wu et al., 2014; Yao et al., 2016

Sugawara et al., 2012; Yang et al., 2012; Zhao et al., 2013; Wu et al., 2014; Sato et al., 2014; Yao et al., 2016 2013; Zeng et al., 2013; Yu et al., 2013; Sampino et al., 2014; Wu et al., 2014
Figure 1 Effects of blastomere biopsy in the short, medium and long term. Schematic overview of the current state of the art in the mouse model. 


\section{Conclusion}

The present review summarizes the current knowledge of the short-, medium- and long-term effects of BB in the mouse model (Fig. 1) and provide the limited information available on TB. What emerges is that, even from the animal model, we still have a fragmentary picture of the real risks (or safety) of $\mathrm{BB}$, and there is no information on the potential side effect (if any) of TB. Thus, much research is still necessary to provide a complete and satisfactory picture of the potential embryo biopsyinduced risk(s) and of the mechanisms behind the onset of developmental defects. Researches focused on BB are still important, as they will provide indication on the potential long-term risk that could affect the already born children in adulthood and/or their progeny, while studies focused on TB are necessary to provide useful information on an invasive procedure whose application is growing nowadays. Through focused investigations, it will be possible to improve not only our scientific knowledge in the field of developmental/reproductive biology, but also current practices in human assisted reproductive technology, to ultimately prevent and manage the onset of diseases in modern society.

\section{Declaration of interest}

The authors declare no conflicts of interest. The contents of this publication are the sole responsibility of the Institute of Genetics and Animal Breeding of the Polish Academy of Sciences and do not necessarily reflect the opinion of the European Union.

\section{Funding}

This work was supported by the National Science Centre of Poland (GA 2015/19/D/NZ4/03696 to FZ), by the KNOW Leading National Research Centre - Scientific Consortium 'Healthy Animal - Safe Food' (GA KNOW/IGHZ/RPB/ WEW/2016/03 to FZ) and by the European Union's Horizon 2020 Research and Innovation Programme (GA 692185 to GEP).

\section{Author contribution statement}

F Z conceived and prepared the manuscript; $R$ A contributed to the manuscript and figure preparation; A A contribute to bibliography research and G E P contributed to the intellectual content and edited the final version of the manuscript.

\section{References}

Banerjee I, Shevlin M, Taranissi M, Thornhill A, Abdalla H, Ozturk O, Barnes J \& Sutcliffe A 2008 Health of children conceived after preimplantation genetic diagnosis: a preliminary outcome study. Reproducitve Biomedicine Online 16 376-381. (doi:10.1016/S14726483(10)60599-8)

Bar-El L, Kalma Y, Malcov M, Schwartz T, Raviv S, Cohen T, Amir H, Cohen Y, Reches A, Amit A et al. 2016 Blastomere biopsy for PGD delays embryo compaction and blastulation: a time-lapse microscopic analysis. Journal of Assisted Reproduction and Genetics 33 1449-1457. (doi:10.1007/s10815-016-0813-2)

Barker DJ 2007 The origins of the developmental origins theory. Journal of Internal Medicine 261 412-417. (doi:10.1111/j.13652796.2007.01809.x)

Carson SA, Gentry WL, Smith AL \& Buster JE. 1993 Trophectoderm microbiopsy in murine blastocysts: comparison of four methods. Journal of Assisted Reproduction and Genetics 10 427-433. (doi:10.1007/ BF01228093)

Cimadomo D, Capalbo A, Ubaldi FM, Scarica C, Palagiano A, Canipari R \& Rienzi L 2016 The impact of biopsy on human embryo developmental potential during preimplantation genetic diagnosis. Biomed Research International 2016 Article ID: 7193075. (doi:10.1155/2016/7193075)

Cui KH, Pannall P, Cates G \& Matthews CD 1993 Blood analysis of mice born following single-cell embryo biopsy. Human Reproduction 8 1906-1909. (doi:10.1093/oxfordjournals.humrep.a137958)

Cui KH, Barua R \& Matthews CD 1994 Histopathological analysis of mice born following single cell embryo biopsy. Human Reproduction $\mathbf{9}$ 1146-1152. (doi:10.1093/oxfordjournals.humrep.a138648)

De Rycke M, Belva F, Goossens V, Moutou C, SenGupta SB, TraegerSynodinos J \& Coonen E 2015 ESHRE PGD Consortium data collection XIII: cycles from January to December 2010 with pregnancy follow-up to October 2011. Human Reproduction 30 1763-1789. (doi:10.1093/ humrep/dev122)

Desmyttere S, De Rycke M, Staessen C, Liebaers I, De Schrijver F, Verpoest W, Haentjens P \& Bonduelle M 2012 Neonatal follow-up of 995 consecutively born children after embryo biopsy for PGD. Human Reproduction 27 288-293. (doi:10.1093/humrep/der360)

Duncan FE, Stein P, Williams CJ \& Schultz RM 2009 The effect of blastomere biopsy on preimplantation mouse embryo development and global gene expression. Fertility and Sterility 91 1462-1465. (doi:10.1016/j. fertnstert.2008.07.1710)

Gentry WL \& Critser ES 1995 Growth of mouse pups derived from biopsied blastocysts. Obstetrics and Gynecology 85 1003-1006. (doi:10.1016/0029-7844(95)00064-X)

Harper JC, Wilton L, Traeger-Synodinos J, Goossens V, Moutou C, SenGupta SB, Pehlivan Budak T, Renwick P, De Rycke M, Geraedts JP et al. 2012 The ESHRE PGD Consortium: 10 years of data collection. Human Reproduction Update 18 234-247. (doi:10.1093/humupd/ dmr052)

Lucas E 2013 Epigenetic effects on the embryo as a result of periconceptional environment and assisted reproduction technology. Reproductive BioMedicine Online 27 477-485. (doi:10.1016/j. rbmo.2013.06.003)

Middelburg KJ, van der Heide $\mathbf{M}$, Houtzager B, Jongbloed-Pereboom $\mathbf{M}$, Fidler V, Bos AF, Kok J, Hadders-Algra M \& PGS Follow-up Study Group 2011 Mental, psychomotor, neurologic, and behavioral outcomes of 2-year-old children born after preimplantation genetic screening: followup of a randomized controlled trial. Fertility and Sterility 96 165-169. (doi:10.1016/j.fertnstert.2011.04.081)

Moutou C, Goossens V, Coonen E, De Rycke M, Kokkali G, Renwick P, SenGupta SB, Vesela K \& Traeger-Synodinos J 2014 ESHRE PGD Consortium data collection XII: cycles from January to December 2009 with pregnancy follow-up to October 2010. Human Reproduction 29 880-903. (doi:10.1093/humrep/deu012)

Monk M, Handyside A, Hardy K \& Whittingham D 1987 Preimplantation diagnosis of deficiency of hypoxanthine phosphoribosyl transferase in a mouse model for Lesch-Nyhan syndrome. Lancet 2 423-425. (doi:10.1016/S0140-6736(87)90959-7)

Pierce KE, Michalopoulos J, Kiessling AA, Seibel MM \& Zilberstein M 1997 Preimplantation development of mouse and human embryos biopsied at cleavage stages using a modified displacement technique. Human Reproduction 12 351-356. (doi:10.1093/humrep/12.2.351)

Piotrowska-Nitsche K, Perea-Gomez A, Haraguchi S \& Zernicka-Goetz M 2005 Four-cell stage mouse blastomeres have different developmental properties. Development 132 479-490. (doi:10.1242/dev.01602)

Sampino S, Zacchini F, Swiergiel AH, Modlinski AJ, Loi P \& Ptak GE 2014 Effects of blastomere biopsy on post-natal growth and behavior in mice. Human Reproduction 29 1875-1883. (doi:10.1093/humrep/deu145)

Sato BL, Sugawara A, Ward MA \& Collier AC 2014 Single blastomere removal from murine embryos is associated with activation of matrix metalloproteinases and Janus kinase/signal transducers and activators 
of transcription pathways of placental inflammation. Molecular Human Reproduction 20 1247-1257. (doi:10.1093/molehr/gau072)

Sugawara A, Sato B, Bal E, Collier AC \& Ward MA 2012 Blastomere removal from cleavage-stage mouse embryos alters steroid metabolism during pregnancy. Biology of Reproduction 87 1-9. (doi:10.1093/ biolreprod/87.s1.1)

Sunkara SK, Antonisamy B, Selliah HY \& Kamath MS 2016 Preterm birth and low birth weight following preimplantation genetic diagnosis: analysis of 88010 singleton live births following PGD and IVF cycles. Human Reproduction 32 432-438. (doi:10.1093/humrep/dew317)

Takeuchi K, Sandow BA, Morsy M, Kaufmann RA, Beebe SJ \& Hodgen GD 1992 Preclinical models for human pre-embryo biopsy and genetic diagnosis. I. Efficiency and normalcy of mouse pre-embryo development after different biopsy techniques. Fertility and Sterility 57 425-430.

Tang F, Barbacioru C, Nordman E, Bao S, Lee C, Wang X, Tuch BB, Heard E, Lao K \& Surani MA 2011 Deterministic and stochastic allele specific gene expression in single mouse blastomeres. PLOS ONE 6 e21208. (doi:10.1371/journal.pone.0021208)

Tarkowski AK, Suwinska A, Czolowska R \& Ozdzenski W 2010 Individual blastomeres of 16- and 32-cell mouse embryos are able to develop into foetuses and mice. Developmental Biology 348 190-198. (doi:10.1016/j. ydbio.2010.09.022)

Taskin AC, Akkoc T, Sagirkaya H, Bagis H \& Arat S 2016 Comparison of the development of mouse embryos manipulated with different biopsy techniques. Turkish Journal of Veterinary and Animal Sciences $\mathbf{4 0}$ 157-162. (doi:10.3906/vet-1506-63)

Terada Y, Ugajin T, Hasegawa H, Nabeshima H \& Yaegashi N 2009 Different embryonic development after blastomere biopsy for preimplantation genetic diagnosis, observed by time-lapse imaging. Fertility and Sterility 92 1470-1471. (doi:10.1016/j.fertnstert.2009.03.009)

Thomaidis L, Kitsiou-Tzeli S, Critselis E, Drandakis H, Touliatou V, Mantoudis S, Leze E, Destouni A, Traeger-Synodinos J, Kafetzis D et al. 2012 Psychomotor development of children born after preimplantation genetic diagnosis and parental stress evaluation. World Journal of Pediatrics 8 309-316. (doi:10.1007/s12519-012-0374-0)

Thompson Jeremy G, Mitchell Megan \& Kind Karen L 2006 Embryo culture and long-term consequences. Reproduction, Fertility and Development 19 43-52. (doi:10.1071/RD06129)

Thornburg KL, O'Tierney PF \& Louey S 2010 Review: the placenta is a programming agent for cardiovascular disease. Placenta 31 S54-S59. (doi:10.1016/j.placenta.2010.01.002)

Torres-Padilla ME, Parfitt DE, Kouzarides T \& Zernicka-Goetz M 2007 Histone arginine methylation regulates pluripotency in the early mouse embryo. Nature 445 214-218. (doi:10.1038/nature05458)

Ugajin T, Terada $Y$, Hasegawa $H$, Velayo $C L$, Nabeshima $H$ \& Yaegashi $N$ 2010 Aberrant behavior of mouse embryo development after blastomere biopsy as observed through time-lapse cinematography. Fertility and Sterility 93 2723-2728. (doi:10.1016/j.fertnstert.2009.06.056)

Wilton LJ \& Trounson AO 1989 Biopsy of preimplantation mouse embryos: development of micromanipulated embryos and proliferation of single blastomeres in vitro. Biology of Reproduction 40 145-152. (doi:10.1095/ biolreprod40.1.145)

Winter C, Van Acker F, Bonduelle M, Desmyttere S \& Nekkebroeck J 2015 Psychosocial development of full term singletons, born after preimplantation genetic diagnosis (PGD) at preschool age and family functioning: a prospective case-controlled study and multi-informant approach. Human Reproduction 30 1122-1136. (doi:10.1093/humrep/ dev036)

Wu Y, Lu Z, Yang Y, Dong G, Yu Y, Cui Y, Tong M, Wang L, Zhou Z, Zhu H et al. 2014 Blastomere biopsy influences epigenetic reprogramming during early embryo development, which impacts neural development and function in resulting mice. Cellular and Molecular Life Science 71 1761-1774. (doi:10.1007/s00018-013-1466-2)

Yang Y, Zeng Y, Lv Z, Wan R, Tong M, Zhu H, Wang L, Zhou Z, Zhou Q \& Sha J 2012 Abnormal development at early postimplantation stage in mouse embryos after preimplantation genetic diagnosis. Anatomical Record 295 1128-1133. (doi:10.1002/ar.22456)

Yao Q, Chen L, Liang Y, Sui L, Guo L, Zhou J, Fan K, Jing J, Zhang Y \& Yao B 2016 Blastomere removal from cleavage-stage mouse embryos alters placental function, which is associated with placental oxidative stress and inflammation. Scientific Reports 6 25023. (doi:10.1038/ srep25023)

Yu Y, Wu J, Fan Y, Lv Z, Guo X, Zhao C, Zhou R, Zhang Z, Wang F, Xiao M et al. 2009 Evaluation of blastomere biopsy using a mouse model indicates the potential high risk of neurodegenerative disorders in the offspring. Molecular and Cellular Proteomics 8 1490-1500. (doi:10.1074/mcp. M800273-MCP200)

Yu Y, Zhao Y, Li R, Li L, Zhao H, Li M, Sha J, Zhou Q \& Qiao J 2013 Assessment of the risk of blastomere biopsy during preimplantation genetic diagnosis in a mouse model: reducing female ovary function with an increase in age by proteomics method. Journal of Proteome Research 12 5475-5486. (doi:10.1021/pr400366j)

Zeng Y, Lv Z, Gu L, Wang L, Zhou Z, Zhu H, Zhou Q \& Sha J 2013 Preimplantation genetic diagnosis (PGD) influences adrenal development and response to cold stress in resulting mice. Cell and Tissue Research 354 729-741. (doi:10.1007/s00441-013-1728-1)

Zhao HC, Zhao Y, Li M, Yan J, Li L, Li R, Liu P, Yu Y \& Qiao J 2013 Aberrant epigenetic modification in murine brain tissues of offspring from preimplantation genetic diagnosis blastomere biopsies. Biology of Reproduction 89 117. (doi:10.1095/biolreprod.113.109926)

Received 2 May 2017

First decision 11 May 2017

Revised manuscript received 14 July 2017

Accepted 29 August 2017 